\title{
Electron drift velocity in silane in strong electric fields determined from rf breakdown curves
}

\author{
V Lisovskiy ${ }^{1}$, J-P Booth ${ }^{2}$, K Landry ${ }^{3}$, D Douai ${ }^{4}$, V Cassagne ${ }^{5}$ and \\ V Yegorenkov ${ }^{6}$ \\ ${ }^{1}$ Department of Physics and Technology, Kharkov National University, Kharkov 61077, \\ Ukraine \\ ${ }^{2}$ Laboratoire de Physique et Technologie des Plasmas, Ecole Polytechnique, \\ Palaiseau 91128, France \\ ${ }^{3}$ Unaxis Displays Division France SAS, 5, Rue Leon Blum, Palaiseau 91120, France \\ ${ }^{4}$ Association Euratom-CEA, Département de Recherches sur la Fusion Contrôlée, \\ CEA Cadarache, Saint Paul lez Durance Cedex F-13108, France \\ ${ }^{5}$ Riber, 31 rue Casimir Périer, Bezons 95873, France \\ ${ }^{6}$ Department of Physics, Kharkov National University, Kharkov 61077, Ukraine \\ E-mail: lisovskiy@yahoo.com
}

Received 14 March 2007, in final form 10 April 2007

Published 18 May 2007

Online at stacks.iop.org/JPhysD/40/3408

\begin{abstract}
We report measurements of the breakdown curves of an rf capacitive discharge in low pressure silane. The electron drift velocity was determined from the locations of the turning point and of the minimum in the breakdown curves in the range $E / p=145-1292 \mathrm{~V} \mathrm{~cm}^{-1} \mathrm{Torr}^{-1}$. We compare our results to values calculated from the published cross-sections in the range $E / p=1-2000 \mathrm{~V} \mathrm{~cm}^{-1} \mathrm{Torr}^{-1}$ and data calculated in other papers and find good agreement.
\end{abstract}

\section{Introduction}

Silane $\left(\mathrm{SiH}_{4}\right)$ is widely used for depositing hydrogenated amorphous and microcrystalline silicon, $\mathrm{SiO}_{2}$ and silicon nitride [1-10]. Therefore a large number of papers are devoted both to experimental studying and to simulating discharge characteristics in $\mathrm{SiH}_{4}$ (see, e.g., [11-17]). In order to use fluid simulations it is necessary to know the electron transport parameters of the gas under study. One of the electron transport parameters, the electron drift velocity $V_{\mathrm{dr}}$, characterizes the conductivity of a weakly ionized gas. However, to our knowledge, the electron drift velocity has been measured and calculated in $\mathrm{SiH}_{4}$ for small values of the reduced electric field $\left(E / p<23 \mathrm{~V} \mathrm{~cm}^{-1} \operatorname{Torr}^{-1}\right.$ [18-22]). The authors of paper [23] calculated the electron drift velocity in strong electric field up to $E / p<1000 \mathrm{~V} \mathrm{~cm}^{-1} \mathrm{Torr}^{-1}$. But it is difficult to estimate the correctness of these results because of the complete absence of experimental data for such strong electric fields.

The well-known techniques of measuring the electron drift velocity (the time-of-flight technique, observation of the optical radiation of a moving electron swarm, the shutter technique and so on) only work for comparatively small reduced fields, $E / p$, because at higher values a self-sustaining discharge is ignited which impedes the measurement. The authors of papers [24-27] have used a novel method for determining the electron drift velocity from the location of the turning point in the breakdown curves of rf capacitive discharges. To supplement this technique, the authors of papers $[28,29]$ proposed a new technique for determining $V_{\mathrm{dr}}$ from the location of the minimum in the rf breakdown curve, and applied both of them to argon, ammonia, nitrogen, hydrogen, oxygen [28] and $\mathrm{N}_{2} \mathrm{O}$ [29]. Whereas conventional techniques become inapplicable after the ignition of the selfsustaining discharge, these two methods are actually based on discharge ignition, allowing measurements of $V_{\mathrm{dr}}$ in strong electric fields.

In this paper we have used these techniques to determine the electron drift velocity in $\mathrm{SiH}_{4}$. Measurements were made in the range $E / p=145-1292 \mathrm{~V} \mathrm{~cm}^{-1} \mathrm{Torr}^{-1}$. With the help of the Bolsig numerical code and published cross-sections [20,21] we have calculated the electron transport parameters in $\mathrm{SiH}_{4}$ in the range $E / p=1-2000 \mathrm{~V} \mathrm{~cm}^{-1} \mathrm{Torr}^{-1}$, and the drift 


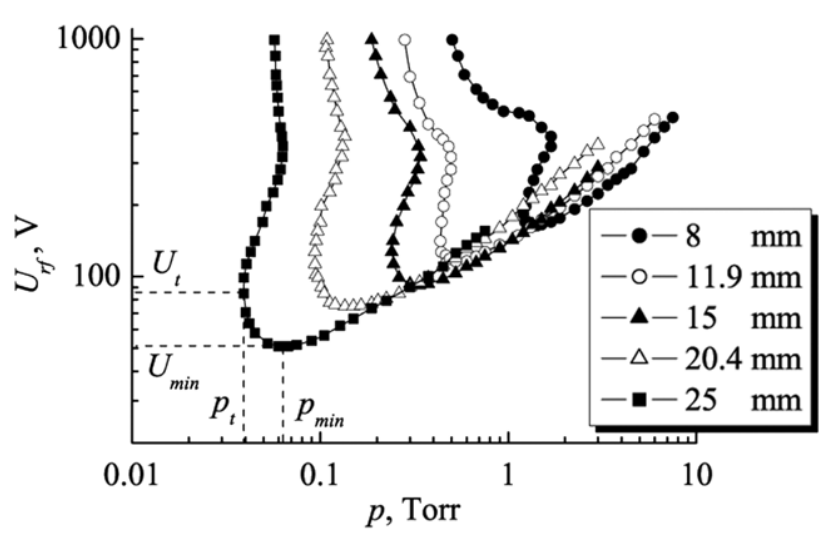

Figure 1. The rf discharge breakdown curves in $\mathrm{SiH}_{4}$ for different inter-electrode gap values.

velocity values obtained from our experiment agree well with the calculated values.

\section{Experimental setup}

The rf discharge was ignited in $\mathrm{SiH}_{4}$ over the pressure range $p \approx 0.03-7.5$ Torr with $\mathrm{rf}$ field frequency $f=13.56 \mathrm{MHz}$. The distance between the flat circular aluminium electrodes (143 mm in diameter) was varied over the range $L=8-25 \mathrm{~mm}$. The rf voltage (amplitude $U_{\mathrm{rf}}<1000 \mathrm{~V}$ ) was fed to one of the electrodes, while the other was grounded. The electrodes were located inside a fused silica tube with an inner diameter of $145 \mathrm{~mm}$. The gas was supplied through small orifices in the powered electrode and then pumped out via the gap between the second electrode and the wall of the fused silica tube.

The gas pressure was monitored with 10 and 1000 Torr capacitive manometers (MKS Instruments). The gas flow was fixed with a mass flow controller to $5 \mathrm{sccm}$, and the pressure regulated by throttling the outlet to the pump. The rf voltage was measured with an rf current-voltage probe (Advanced Energy Z'SCAN).

We used the technique proposed by Levitskii [30] to measure the rf breakdown curves. Near and at the high pressure side of the minimum in the breakdown curve the $\mathrm{SiH}_{4}$ pressure was fixed before slowly increasing the rf voltage until gas breakdown occurs. At the low pressure side of the minimum the curve may be multi-valued, i.e. the curve turns around and breakdown occurs at two different values of the rf voltage. Therefore in this range we first decreased the $\mathrm{SiH}_{4}$ pressure, then fixed the rf voltage value and only then increased the $\mathrm{SiH}_{4}$ pressure slowly until discharge ignition occurred. When discharge ignition occurs the rf voltage decreases sharply, and a glow appears between the electrodes indicating the onset of gas breakdown. The uncertainty in the measured breakdown voltages did not exceed 1-2 V over the whole $U_{\text {rf }}$ range under study.

\section{Experimental results}

We present in figure 1 the rf breakdown curves of $\mathrm{SiH}_{4}$ we registered. The available list of references does not contain papers devoted to the ignition of the rf capacitive discharge in $\mathrm{SiH}_{4}$. Therefore we have no opportunity to compare our results with experimental or calculated data of other authors. Within the rf voltage and pressure ranges we studied that $\mathrm{SiH}_{4}$ rf breakdown curves possess two branches: the Paschen one (to the left of the ignition curve minima, at $\mathrm{rf}$ voltages $U_{\mathrm{rf}}>300 \mathrm{~V}$ ), as well as a diffusion-drift one [25]. Similar to a number of other gases [24-30], diffusion-drift branch of rf breakdown curves of $\mathrm{SiH}_{4}$ has a clearly expressed region of multi-valued dependence of the rf breakdown voltage on gas pressure (at low gas pressure). The reasons for such behaviour of rf breakdown curves are described in detail in papers [24-30], therefore we will not discuss it here.

We need rf breakdown curves to use them for determining the values of the electron drift velocity in $\mathrm{SiH}_{4}$. On finding the coordinates of the turning point $\left(U_{\mathrm{t}}\right.$ and $p_{\mathrm{t}}$, see figure 1$)$, we can determine the value of the electron drift velocity at the point according to the formula

$$
V_{\mathrm{dr}}=L \pi f
$$

where $L$ is the inter-electrode distance, and $f$ is the rf electric field frequency. From $U_{\mathrm{t}}$ and $p_{\mathrm{t}}$ we determine the value of the reduced electric field $(E / p)_{\mathrm{t}}=U_{\mathrm{t}} /\left(p_{\mathrm{t}} L\right)$, to which the electron drift velocity thus measured corresponds. Equation (1) for drift velocity determination from the turning point of the rf breakdown curve is universal, i.e. it can be applied for any value of the reduced field $E / p$, any frequency of the rf $f$. Obviously, a breakdown curve should have a turning point for this technique to be applicable. We can use equation (1) for low $E / p$ values when $V_{\mathrm{dr}} \propto \frac{E}{p}$, as well as for $E / p$ values when $V_{\mathrm{dr}} \propto \sqrt{\frac{E}{p}}$.

The coordinates of the rf breakdown curve minimum $\left(U_{\min }\right.$ and $\left.p_{\min }\right)$ and, respectively, the value of the reduced field $(E / p)_{\min }$ allow us to find another value of the electron drift velocity according to the formula [28]

$$
V_{\mathrm{dr}}=L \cdot \pi \cdot f \cdot \frac{B-\frac{1}{\sqrt{2}}\left(\frac{E}{p}\right)_{\min }}{B+\frac{1}{\sqrt{2}}\left(\frac{E}{p}\right)_{\min }} .
$$

A disadvantage of this technique is that it depends on knowledge of the constant $B$, which is related to the first Townsend coefficient $\alpha$ by the following expression:

$$
\alpha=A \cdot p \cdot \exp \left(-\frac{B}{E_{\mathrm{eff}} / p}\right),
$$

where $E_{\text {eff }}$ is the effective electric field strength, $E_{\text {eff }}=$ $E_{\mathrm{rf}} / \sqrt{2}, A$ and $B$ are constants depending on the gas species. Therefore this technique can only be applied to gases for which this first Townsend coefficient is known. Within the range of the reduced field $E / p=130-600 \mathrm{~V} \mathrm{~cm}^{-1} \mathrm{Torr}^{-1}$ the curve for the first Townsend coefficient $\alpha$ we obtained with the Bolsig code, may be approximated with formula (3) with the values of the constants $A=22 \mathrm{~cm}^{-1}$ Torr $^{-1}, B=530 \mathrm{~V} \mathrm{~cm}^{-1}$ Torr $^{-1}$.

Thus, from a single experimental $\mathrm{rf}$ breakdown curve we can get two values of the electron drift velocity-from the turning point and minimum (under condition that the turning point is well expressed). This technique for electron drift velocity determination is described in detail in $[28,29]$. 


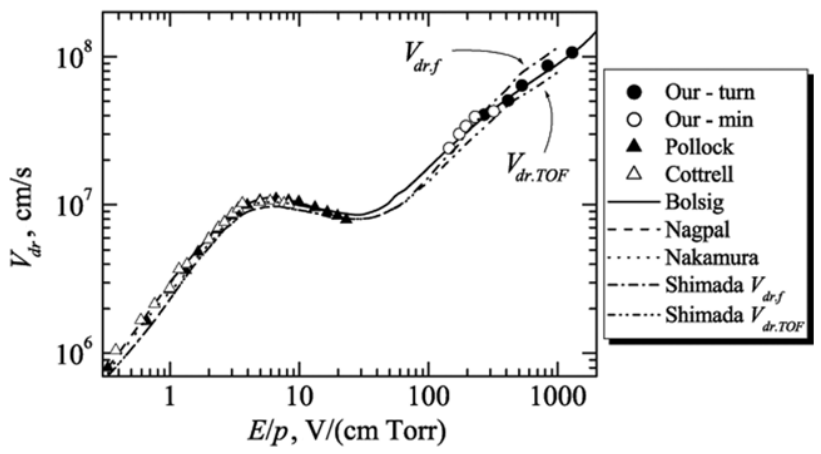

Figure 2. Electron drift velocity in $\mathrm{SiH}_{4}$ against $E / p$. Solid circles are for our measured data (from the turning points), empty circles are for our measured data (from the minima), solid triangles are for the experimental data from [18], empty triangles are for the experimental data from [19], the solid curve presents the data calculated with the Bolsig code, the dashed curve presents the calculation data from [22], the dotted curve presents the calculation data from [20,21], the dash-dotted curve and the dash-dotted-dotted curve present the calculation data from [23] (flux drift velocity $V_{\text {drf }}$ and the centre of mass or time-of-flight drift velocity $V_{\mathrm{drTOF}}$, respectively).

On the whole, we registered five rf breakdown curves of $\mathrm{SiH}_{4}$ and, consequently, got ten values of the electron drift velocity for different $E / p$. The results we obtained are presented in figure 2 together with experimental data from [18, 19], as well as calculated data from [20-23]. As we have already said, experimental data of $[18,19]$, as well as calculated ones from [20-22] relate to the range of the reduced field $E / p<23 \mathrm{~V} \mathrm{~cm}^{-1} \mathrm{Torr}^{-1}$. Our data are obtained in the range $E / p=145-1292 \mathrm{~V} \mathrm{~cm}^{-1} \mathrm{Torr}^{-1}$. Therefore it has no sense to compare our results with the data from [18-22] (see figure 2). The authors of paper [23] employed a direct numerical procedure to solve the Boltzmann equation. They got the values of the electron drift velocity (flux drift velocity $V_{\mathrm{drf}}$ and the centre of mass or time of flight drift velocity $\left.V_{\mathrm{drTOF}}\right)$ within the range of the reduced field $E / p<$ $1000 \mathrm{~V} \mathrm{~cm}^{-1}$ Torr $^{-1}$. As seen from figure 2, our measured data are in reasonable agreement with calculated ones [23].

We also calculated the electron drift velocity in $\mathrm{SiH}_{4}$ with the Bolsig code (www.siglo-kinema.com/bolsig.htm) in the range $E / p=1-2000 \mathrm{~V} \mathrm{~cm}^{-1} \mathrm{Torr}^{-1}$. (Bolsig is a code for the numerical solution of the Boltzmann equation for electrons in weakly ionized gases and in steady-state, uniform fields. This code was designed to generate electron transport data in pure gases or gas mixtures.) We used the cross-section data on electron collisions with $\mathrm{SiH}_{4}$, obtained by authors of [20,21]. The results of our calculations are also shown in figure 2, and they are in good agreement with the values of the electron drift velocity we obtained.

\section{Conclusions}

We have determined the electron drift velocity in $\mathrm{SiH}_{4}$ from the location of the turning points and minima of the breakdown curves of rf capacitive gas discharges within the range $E / p=145-1292 \mathrm{~V} \mathrm{~cm}^{-1} \mathrm{Torr}^{-1}$. With the help of the numerical Bolsig code and published cross-sections we have calculated the electron drift velocity $V_{\mathrm{dr}}$ in $\mathrm{SiH}_{4}$ in the range $E / p=1-2000 \mathrm{~V} \mathrm{~cm}^{-1} \mathrm{Torr}^{-1}$. The values of the drift velocity obtained from the experiment are in good agreement with the calculated data.

\section{Acknowledgment}

The authors express their gratitude to the Unaxis France Displays Division, Palaiseau, France, for their financial support and for the equipment used in this study.

\section{References}

[1] Collins R W 1986 J. Vac. Sci. Technol. A 4514

[2] Mataras D, Cavadias S and Rapakoulias D 1993 J. Vac. Sci. Technol. A 11664

[3] Srinivasan E and Parsons G N 1997 J. Appl. Phys. 812847

[4] Chowdhury A I, Klein T M, Anderson T M and Parsons G N 1998 J. Vac. Sci. Technol. A 161852

[5] Leroy O, Gousset G, Alves L L, Perrin J and Jolly J 1998 Plasma Sources Sci. Technol. 7348

[6] Biebericher A C W, Bezemer J and Weg W F 2000 Appl. Phys. Lett. 762002

[7] Hadjadj A, Beorchia A, Boufendi L, Huet S and Roca I Cabarrocas P $2001 \mathrm{~J}$. Vac. Sci. Technol. A 19124

[8] Viera G, Huet S, Bertran E and Boufendi L 2001 J. Appl. Phys. 904272

[9] Chen F F 1995 Phys. Plasmas 22164

[10] Amanatides E, Hammad A, Katsia E and Mataras D 2005 J. Appl. Phys. 97073303

[11] Makabe T, Tochikubo F and Nishimura M 1990 Phys. Rev. A 423674

[12] Bohm Ch and Perrin J 1991 J. Phys. D: Appl. Phys. 24865

[13] Boeuf J P and Belenguer Ph 1992 J. Appl. Phys. 714751

[14] Perrine J 1993 J. Phys. D: Appl. Phys. 261662

[15] Fridman A A, Boufendi L, Hbid T, Potapkin B V and Bouchoule A 1996 J. Appl. Phys. 791303

[16] Kim D-J and Kim K-S 1997 Japan. J. Appl. Phys. 364989

[17] Nienhuis G J, Goedheer W J, Hamers E AG, van Sark W G J H M and Bezemer J 1997 J. Appl. Phys. 822060

[18] Pollock W J 1968 Trans. Faraday Soc. 642919

[19] Cottrell T L and Walker I C 1965 Trans. Faraday Soc. 611585

[20] Kurachi M and Nakamura Y 1989 J. Phys. D: Appl. Phys. 22107

[21] Kurachi M and Nakamura Y 1991 IEEE Trans. Plasma Sci. 19262

[22] Nagpal R and Garscadden A 1994 A new collision cross section set for silane Gaseous Dielectrics VII ed L G Christophorou and D R James (New York: Plenum) pp 39-45

[23] Shimada T, Nakamura Y, Petrovich Z Lj and Makabe T 2003 J. Phys. D: Appl. Phys. 361936

[24] Lisovskiy V A 1998 Tech. Phys. Lett. 24308

[25] Lisovskiy V A and Yegorenkov V D 1998 J. Phys. D: Appl. Phys. 313349

[26] Lisovskiy V A and Yegorenkov V D 1999 J. Phys. D: Appl. Phys. 322645

[27] Lisovskiy V, Martins S, Landry K, Douai D, Booth J-P and Cassagne V 2005 J. Phys. D: Appl. Phys. 38872

[28] Lisovskiy V, Booth J-P, Landry K, Douai D, Cassagne V and Yegorenkov V 2006 J. Phys. D: Appl. Phys. 39660

[29] Lisovskiy V, Booth J-P, Landry K, Douai D, Cassagne V and Yegorenkov V 2006 J. Phys. D: Appl. Phys. 391866

[30] Levitskii S M 1957 Sov. Phys.-Tech. Phys. 2887 